J. T. Gresser

Nagoya Math. J.

Vol. 51 (1973), 185-189

\title{
RESTRICTED PRINCIPAL CLUSTER SETS OF A CERTAIN HOLOMORPHIC FUNCTION
}

\author{
JOHN T. GRESSER
}

Let $D$ be the open unit disk and let $K$ be the unit circle. We say that $\alpha$ is an arc at $\zeta \in K$ if $\alpha$ is contained in $D$ and is the image of a continuous function $z=z(t)(0 \leq t<1)$ such that $z(t) \rightarrow \zeta$ as $t \rightarrow 1$. We call $\alpha$ a segment as $\zeta$ if the function $z=z(t)$ is linear in $t$. If $P$ is a property which is meaningful for each point of $K$, we say that nearly every point of $K$ has property $P$ if the exceptional set is a set of first Baire category in $K$. We assume that the reader is familiar with the rudiments of cluster set theory, and in particular with the terms ambiguous point, Meier point, and Plessner point of a function (cf. [4] or [7]). If $f$ is a function which maps $D$ into the Riemann sphere, if $\zeta \in K$ and $\alpha$ is an arc at $\zeta$, then $C(f, \zeta, \alpha)$ will denote the arc-cluster set of $f$ at $\zeta$ along $\alpha$. The principal cluster set of $f$ at $\zeta$ is defined to be the set

$$
\Pi(f, \zeta)=\bigcap_{\alpha} C(f, \zeta, \alpha),
$$

where $\alpha$ ranges over all arcs of $\zeta$. The nontangential principal cluster set of $f$ at $\zeta$ is defined to be the set

$$
\Pi^{*}(f, \zeta)=\bigcap_{\alpha} C(f, \zeta, \alpha),
$$

where $\alpha$ ranges over all arcs at $\zeta$ for which there is a Stolz angle at $\zeta$ containing $\alpha$. Finally, if $\Delta$ is a Stolz angle at $\zeta$, we define the set

$$
\Pi_{\Delta}(f, \zeta)=\bigcap_{\alpha} C(f, \zeta, \alpha),
$$

where $\alpha$ ranges over all arcs at $\zeta$ such that $\alpha \subseteq \Delta$.

Let $\Delta$ be a Stolz angle at $\zeta_{0}=1$, and for each $\zeta \in K$ let $\Delta(\zeta)$ be the Stolz angle at $\zeta$ which is obtained by rotating $\Delta$ about the origin. We

Received March 17, 1971. 
mention as background for this paper two well-known results by E. F. Collingwood. Let $f$ be an arbitrary complex-valued function defined in D. Then

$$
C_{\Delta(\zeta)}(f, \zeta)=C(f, \zeta)
$$

for nearly every point $\zeta \in K$, and

$$
C_{B}(f, \zeta)=C(f, \zeta)
$$

for all but a countable number of points $\zeta \in K$. Here $C_{B}(f, \zeta)$ denotes the boundary cluster set of $f$ at $\zeta$ (cf. [4, pp. 80, 82]). More recently, using analogous definitions and techniques, the boundary principal cluster set of $f$ at $\zeta$, denoted by $B \Pi(f, \zeta)$, has been investigated [5]. This set describes the behavior of $\Pi(f, \xi)$ at points $\xi$ which are near and distinct from $\zeta$. It has been shown that if $f$ is continuous in $D$, then

$$
B \Pi(f, \zeta)=\Pi(f, \zeta)
$$

for nearly every point $\zeta \in K$ [5, Theorem 9]. This naturally suggests the question of whether for a continuous function $f$ in $D$ it follows that

$$
\Pi_{\Delta(\zeta)}(f, \zeta)=\Pi(f, \zeta)
$$

for nearly every point $\zeta \in K$. The purpose of this paper is to answer this question in the negative, even for a holomorphic function; we show, in fact, that an even stronger inequality is possible. We use the symbol $\subset$ in the sequel to denote proper set inclusion.

THEOREM. Let $\Delta$ be a Stolz angle at $\zeta_{0}=1$. Then there exists $a$ holomorphic function $f$ in $D$ such that

$$
\Pi^{*}(f, \zeta) \subset \Pi_{\Delta(\zeta)}(f, \zeta)
$$

for nearly every point $\zeta \in K$.

Proof. Our construction is a modification of a method presented by F. Bagemihl in [2]. If $\rho$ is a segment at $\zeta$, we let $\theta(\rho)$ denote the angle $(0<\theta(\rho)<\pi)$ between $\rho$ and the forward tangent to the unit circle at $\zeta$, and we let $\ell(\rho)$ denote the length of $\rho$.

For each ternary fraction

$$
t=0 \cdot t_{1} t_{2} t_{3} \cdots
$$

where each $t_{j}$ is zero or two, we denote by 


$$
b(t)=0 \cdot b_{1} b_{2} b_{3} \cdots
$$

the binary fraction such that for each $j=1,2,3, \ldots$

$$
b_{j}=\left\{\begin{array}{lll}
0 & \text { if } & t_{j}=0 \\
1 & \text { if } & t_{j}=2 .
\end{array}\right.
$$

The set $T$ of all such ternary fractions is the Cantor "middle thirds" set, and the set of all corresponding binary fractions is the closed unit interval. We set $T^{*}=T-\{1\}$, and for each $t \in T^{*}$ we let

$$
\zeta_{t}=e^{2 \pi i b(t)} \text {. }
$$

We let $\theta_{0}$ be any fixed number $\left(0<\theta_{0}<\pi\right)$ such that $\theta(\rho)>\theta_{0}$ for every segment $\rho$ at $\zeta_{0}=1$ which is contained in $\Delta$. Then for each $t \in T^{*}$ we let $\rho_{t}$ be the segment at $\zeta_{t}$ defined by

$$
\theta\left(\rho_{t}\right)=(1-t) \theta_{0} / 4 \text {, and } \ell\left(\rho_{t}\right)=(1-\sin (\pi t / 2)) \sin \left(\theta_{0} / 4\right) .
$$

Since $\ell\left(\rho_{t}\right)<\sin \left(\theta\left(\rho_{t}\right)\right)$ for $t \in T^{*}$, it follows that the line perpendicular to $\rho_{t}$ and passing through the origin does not intersect $\rho_{t}$. In addition, $\theta\left(\rho_{t}\right)$ is a decreasing function of $t$ which is always less than $\pi / 4$. These observations easily imply that for each $t \in T^{*}$ the segments in the collection

$$
\left\{\rho_{s}: s \in T^{*}, s \geq t, \quad \text { and } \arg \zeta_{t} \leq \arg \zeta_{s} \leq \arg \zeta_{t}+\pi / 2\right\}
$$

are mutually nonintersecting. Thus in order to establish that the segments in the collection

$$
P=\left\{\rho_{t}: t \in T^{*}\right\}
$$

are mutually nonintersecting, it suffices to show that for each $t \in T^{*}$ such that $\zeta_{t}$ lies in the fourth quadrant, the corresponding segment $\rho_{t}$ lies in the lower half of $D$. This follows from the observation that for each $t \in T^{*}$ with $t>7 / 9$ we have $t>b(t)>3 / 4$ and hence

$$
\ell\left(\rho_{t}\right)<1-\sin (\pi b(t) / 2)<1-\cos (2 \pi b(t))<|\sin (2 \pi b(t))| .
$$

There is a countably dense subset $E$ of $K$ such that for each $\zeta \in E$ there are two segments, say $\alpha_{\zeta}$ and $\beta_{\zeta}$, at $\zeta$ belonging to $P$, while for each $\zeta \in K-E$ there is exactly one segment at $\zeta$ belonging to $P$. For each $\zeta \in E$ we let $\tau_{\zeta}$ be the segment at $\zeta$ defined by

$$
\theta\left(\tau_{\zeta}\right)=(1 / 2)\left(\theta\left(\alpha_{\zeta}\right)+\theta\left(\beta_{\zeta}\right)\right) \text { and } \ell\left(\tau_{\zeta}\right)=(1 / 2)\left(\ell\left(\alpha_{\zeta}\right)+\ell\left(\beta_{\zeta}\right)\right),
$$


and we let

$$
Q=\left\{\tau_{\zeta}: \zeta \in E\right\}
$$

It follows easily from [3, Theorem 1, p. 187-8] that there is a holomorphic function $f$ defined in $D$ such that for every $\rho \in P$

$$
f(z) \longrightarrow 0 \text { as }|z| \longrightarrow 1 \text { along } \rho,
$$

and for every $\tau \in Q$

$$
f(z) \longrightarrow \infty \text { as }|z| \longrightarrow 1 \text { along } \tau \text {. }
$$

Because $E$, and $K-E$ are both dense in $K$, we have that $0, \infty \in C(f, \zeta)$ for every $\zeta \in K$, and hence no point of $K$ is a Meier point of $f$. Therefore, nearly every point of $K$ is a Plessner point of $f[6$, Theorem 6 , p. 330]. We will show that

$$
\infty \in \Pi_{\Delta(\zeta)}(f, \zeta)
$$

for every Plessner point $\zeta$ which is not an ambiguous point of $f$. Since there are at most countably many ambiguous points of $f[1$, Theorem 2, p. 380], and since $\Pi^{*}(f, \zeta) \subseteq\{0\}$ for every $\zeta \in K$, the verification of (1) will complete the proof.

Let $\zeta$ be a Plessner point of $f$ which is not an ambiguous point of $f$, and suppose, to the contrary, that $\gamma$ is an arc at $\zeta$ with $\gamma \subseteq \Delta(\zeta)$ such that $\infty \notin C(f, \zeta, \gamma)$. If $\rho$ is the segment at $\zeta$ belonging to $P$, then $\infty \notin C(f, \zeta, \rho)$. Since the region bounded by $\gamma, \rho$, and any third convenient arc in $D$ contains a Stolz angle, it follows that the full cluster set of $f$ at $\zeta$ restricted to this subregion is total. By the Gross-Iversen theorem [4, Theorem 5.8, p. 101] it follows that $\infty$ is an asymptotic value of $f$ at $\zeta$, and this contradicts our assumption that $\zeta$ is not an ambiguous point of $f$. Thus (1) is established and the proof is complete.

\section{REFERENCES}

[1] F. Bagemihl, Curvilinear cluster sets of arbitrary functions, Proc. Nat. Acad. Sci., 41 (1955), 379-382.

[2] F. Bagemihl, The chordal and horocyclic principal cluster sets of a certain holomorphic function, Yokohama Math. J., 16 (1968), 11-14.

[ 3 ] F. Bagemihl and W. Seidel, Some boundary properties of analytic functions, Math. Zeitschr., 61 (1954), 186-199.

[4] E. F. Collingwood and A. J. Lohwater, The theory of cluster sets, Cambridge, 1966. 
[5] J. T. Gresser, The local behavior of principal and chordal principal cluster sets, Trans. Amer. Math. Soc., 165 (1972), 323-332.

[6] K. Meier, Uber die Randwerte der meromorphen Funktionen, Math. Ann., 142 (1961), 328-344.

[ 7 ] K. Noshiro, Cluster sets, Berlin, 1960.

Bowling Green State University 